\title{
NUMERICAL MODELING OF UHPFRC TENSILE BEHAVIOR BY A MICROMECHANICS FEM MODEL TAKING INTO ACCOUNT FIBER ORIENTATION
}

\section{THOMAS GUÉNET ${ }^{* \dagger}$, FLORENT BABY*, YOURI DUHAMEL-LABRECQUE ${ }^{\dagger}$, SAMUEL MEULENYZER ${ }^{\ddagger}$, LUCA SORELLI ${ }^{\dagger}$, FRANÇOIS TOUTLEMONDE* ${ }^{*}$ AND SÉBASTIEN BERNARDI ${ }^{\ddagger}$}

\author{
*UPE-IFSTTAR \\ Marne-la-Vallée, France \\ e-mail: thomas.guenet@ifsttar.fr \\ ${ }^{\dagger}$ CRIB, Laval University \\ Québec, QC Canada \\ ${ }_{\ddagger}^{\ddagger}$ LafargeHolcim \\ Paris, France
}

Key words: UHPFRC, micromechanics, smeared crack model, fiber orientation, bending tests

\begin{abstract}
Ultra High Performance Fiber Reinforced Concrete (UHPFRC) structures are emerging in several engineering applications as their outstanding tensile strength and ductility allow engineers to develop new structural concepts and overcome construction limits. Optimization of the UHPFRC fiber ratio, which has significant economic and technical relevance, is critically related to guaranteeing ductile failure modes of such structures and components, including effects of the scatter of local material properties and post-cracking fiber contributions. A micromechanical model of UHPFRC tensile behavior taking into account fiber orientation, fiber volume ratio, and material parameters concerning the fibers and the cementitious matrix has been implemented in a FEM software.

The constitutive law was developed to describe the fiber pullout and the matrix cracking mechanism within a smeared rotating crack framework. We statistically average the orientation of fibers by calibrating the model parameters on results from tomography analysis. The obtained constitutive law is applied to UHPFRC beams with and without reinforcing bars, considering two fiber volume ratios (1\% and $2 \%)$. The numerical model has shown the capacity to grasp the specimens behavior with and without reinforcing bars.
\end{abstract}

\section{INTRODUCTION}

Ultra High Performance Fiber-Reinforced Concrete (UHPFRC) refers to a class of materials with a cementitious matrix, a characteristic compressive strength in excess of $130 \mathrm{MPa}$, and containing fibers in order to achieve a ductile behavior under tension [1]. Fibers avoid brittle failure compared to ordinary concrete. Unfortunately, this behavior severely depends on fiber orientation, which induces difficulties to predict the ductility of civil engineering structures. The $K$-orientation factors have been introduced to cope with this issue in structural design $[2,3]$. However, its determination is rather labored consuming and implementation is associated with a rough discretization of the structure [4]. Indeed, the orientation of fibers depends on shear flow, wall effect, casting meth- 
ods, viscous properties of fresh concrete and eventual vibration [5]. Table 1 shows how fracture energy decreases when fiber orientation diverges from principal stress direction. The values were extracted from tensile and bending tests, and then divided by the maximal measured fracture energy $[7,8]$. As a consequence, parameters such as the geometry of the element to be cast, the rheology of the material and the casting process are essential [6].

Table 1: Energy release rates as a function of fiber orientation relatively to the principal stress orientation corresponding to $0^{\circ}, \mathcal{G} / \mathcal{G}_{\vartheta}=0$

\begin{tabular}{lccccc}
\hline Orientation & $0^{\circ}$ & $30^{\circ}$ & $45^{\circ}$ & $60^{\circ}$ & $90^{\circ}$ \\
\hline Traction [7] & 1 & 0.92 & 0.64 & 0.38 & 0.29 \\
Bending [8] & 1 & 0.66 & 0.32 & 0.11 & 0.07 \\
\hline
\end{tabular}

To improve design requirements of $\mathrm{UH}-$ PFRC elements, fiber orientation with regards of principal stress direction should be taken into account. Thus, an accurate UHPFRC structural analysis can only be achieved by correctly modeling fiber orientation effects, crack growth, hardening and softening behavior.

Several models have been developed with this aim in using micromechanical approaches. These models make it possible to locally consider fiber orientation in structure by describing the pullout mechanism. Fiber orientation was firstly considered either uniform [9], or unidirectional [10]. The uniform case was then enriched using a fiber orientation efficiency factor [11], considering values from pullout tests $[12,13]$. The anisotropic orientation of fiber was also considered to model the hardening uniaxial behavior of UHPFRC [14] thanks to a Gaussian-like distribution function. Another technique consists of modeling orientation effects thanks to an empirical model which provides the UHPFRC uniaxial pre-peak and postpeak tensile behavior from the average fiber orientation measured in specimens or structural elements by 2D image analysis [16].

To model crack propagation of UHPFRC, the constitutive law should eventually be hard- ening depending on material parameters and softening afterwards.

In this paper, following previsions simplified attempt [15], we use a micromechanical based model which takes into account fiber orientation using a bivariate normal-like probability distribution. The model reproduces the hardening of UHPFRC based on the principle of energy release rates and the softening behavior established within the framework of a smeared rotating crack model. The model is able to reproduce fiber orientation effect on results from the literature. It is then applied to a specific experimental campaign to evaluate its efficiency to grasp the behavior of reinforced UHPFRC simple elements where fiber volumetric content and fiber orientation are informed from tomography analysis. Main results are summarized and discussed in the last sections.

\section{THE MICROMECHANIC MODEL}

The stress-strain law of UHPFRC materials can be divided into three parts: elastic, hardening and softening. The hardening behavior may only occur under some conditions in particular when the volume fiber ratio is high enough and if the main fiber orientation is close enough to the principal tensile stress direction.

Micromechanical based models make it possible to identify whether or not a hardening phase will develop considering the usual material parameters related to fibers, to the cementitious matrix and their interactions. Indeed the hardening and softening behaviors are described as functions of the extraction law established from the pullout mechanism of fibers.

\subsection{Pullout mechanism of fibers}

Firstly, the pullout force $(F)$ as to be defined. It can be distinguished between the debonding force $\left(F_{d}\right)$ and the post-debonding force due to friction $\left(F_{p}\right)$. The relationship between the extraction forces with respect to the crack opening displacement $(w)$ is derived [9].

$$
\begin{aligned}
& \text { For } 0<w \leq w_{0}: \\
& F_{d}(w)=\frac{1}{2} \phi_{f} \pi \sqrt{(1+\chi) \tau_{0} E_{f} \phi_{f}} \sqrt{w},
\end{aligned}
$$


and for $w_{0}<w \leq L_{f} / 2$ :

$$
F_{p}(w)=\pi \phi_{f} \tau_{0}(1+\chi)\left(L-w+w_{0}\right),
$$

with

$\chi=\frac{E_{f} v_{f}}{E_{m}\left(1-v_{f}\right)}$ and $w_{0}=\frac{4(1+\chi) \tau_{0} L^{2}}{E_{f} \phi_{f}}$

where $\phi_{f}$ : fiber diameter; $E_{f}$ : fiber elastic modulus ; $E_{m}$ : matrix elastic modulus; $v_{f}$ : volume of fibers; $L$ : embedded length of fiber; $\tau_{0}$ : bond strength of fiber-matrix interface and $w_{0}$ is the crack opening displacement at which the fiber is fully debonded. The debonding effect due to the Poisson's ratio is not considered in the model [9], while the group effect of fibers is indirectly taken into account in the calibration of the fiber bond strength.

Secondly, the cohesive stress across the crack $\left(\sigma_{c}\right)$ is derived from the integration of the fiber pullout forces and the probability $(p)$ that fibers intercept the crack plane [9]. $p$ can be decomposed by the product of $p_{z}$ and $p_{A z, E l}$ which respectively account for the fiber dispersion in space and the fiber orientation defined by azimuth $(A z)$ and elevation $(E l)$.

$$
\begin{aligned}
\sigma_{c}(w) & =\frac{4 v_{f}}{\pi \phi_{f}^{2}} \int_{0}^{2 \pi} \int_{0}^{\frac{\pi}{2}} \int_{0}^{z_{0} \cos E l \cos A z} F(w) p_{z} p_{A z, E l} \mathrm{~d} S \\
\mathrm{~d} S & =\cos E l \mathrm{~d} z \mathrm{~d} E l \mathrm{~d} A z
\end{aligned}
$$

where $z$ is the distance of the centroid of the fiber from crack plan and $p_{z}$ its probability distribution. $p_{z}(z)=2 / L_{f}$ is chosen uniformly distributed in the representative element volume. The integrals in Eq. 4 simply count the number of fibers bridging the crack considering their embedded length and orientation toward the crack plane.

\subsection{Fiber orientation distribution}

The casting process has an important effect on fiber orientation $[6,17]$. In this work we use a general bivariate $\pi$-periodic normal-like probability density function $\left(p_{A z, E l}=p_{\boldsymbol{\mu}, \boldsymbol{\Sigma}}\right)$ to consider the local fiber orientation:

$$
\begin{aligned}
p_{\boldsymbol{\mu}, \boldsymbol{\Sigma}}(\mathbf{x}) & =\frac{1}{2 \pi \sqrt{|\boldsymbol{\Sigma}|}} e^{\left[-\frac{1}{2}(\mathbf{x}-\boldsymbol{\mu})^{t} \boldsymbol{\Sigma}^{-1}(\mathbf{x}-\boldsymbol{\mu})\right]} \\
\boldsymbol{\mu} & =\left(\begin{array}{c}
\mu_{A z} \\
\mu_{E l}
\end{array}\right)
\end{aligned}
$$

where $\mathbf{x}=\langle E l, A z\rangle$ is the vector of random variables elevation and azimuth, $\boldsymbol{\mu}$ is the column-vector representing the center of the function and $\Sigma$ is the covariance matrix ( $\zeta$ is the rotation angle):

$$
\begin{aligned}
\Sigma_{11} & =\left(\sigma_{A z} \cos \zeta\right)^{2}+\left(\sigma_{E l} \sin \zeta\right)^{2} \\
\Sigma_{22} & =\left(\sigma_{A z} \sin \zeta\right)^{2}+\left(\sigma_{E l} \cos \zeta\right)^{2} \\
\Sigma_{12}=\Sigma_{21} & =\left(\sigma_{A z}^{2}-\sigma_{E l}^{2}\right) \sin \zeta \cos \zeta
\end{aligned}
$$

The azimuth and elevation are linked to Cartesian coordinates as shown in Fig. $1 ; \sigma_{A z}$ and $\sigma_{E l}$ are respectively the standard deviation of the azimuth and the elevation.

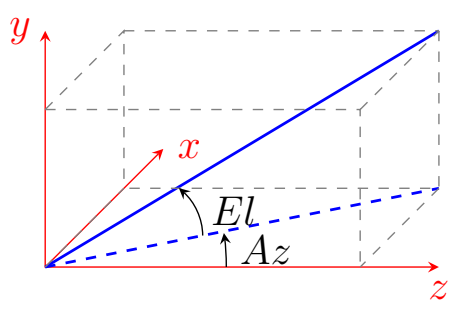

Figure 1: Azimuth and Elevation Coordinates

By simplifying Eq. 4, one can identify the standard orientation factor (defined by Krenchel) following direction $z$. Figure 2 shows values of the orientation factor $\alpha$ in function of $\mu_{E l}\left(\mu_{A z}=0\right)$ and $\sigma_{E l}\left(=\sigma_{A z}\right)$.

$$
\begin{aligned}
\alpha_{z} & =\int_{A z=0}^{2 \pi} \int_{E l=0}^{\frac{\pi}{2}} p_{\boldsymbol{\mu}, \boldsymbol{\Sigma}}(E l, A z) \mathrm{d} S, \\
\mathrm{~d} S & =\cos ^{2} E l \cos A z \mathrm{~d} E l \mathrm{~d} A z .
\end{aligned}
$$

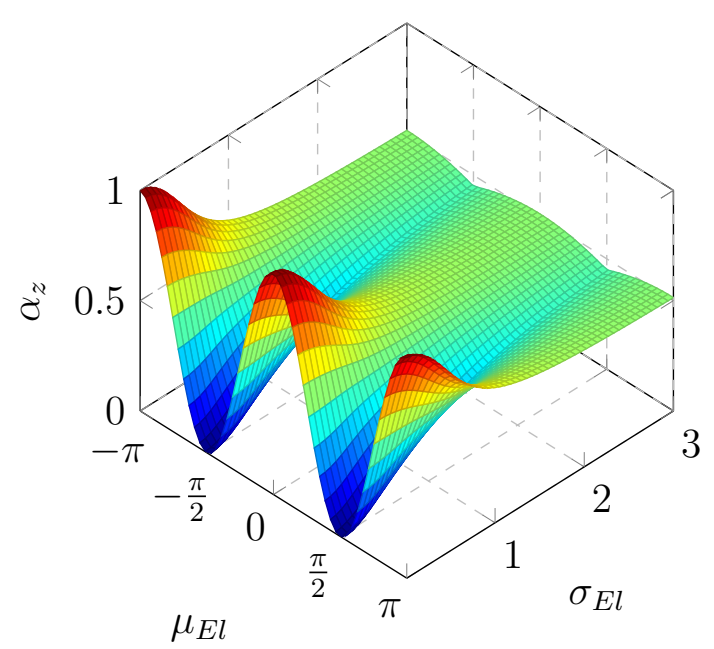

Figure 2: Orientation factor of the model function of parameters $\left(\sigma_{A z}=\sigma_{E l}\right)$ and $\mu_{E l}\left(\mu_{A z}=0\right)$ 
This probability density function is able to reproduce typical values of fiber orientation factors identified in literature [17] $(1 \mathrm{D}, 2 \mathrm{D}$ and 3D uniform orientation distribution). Correspondence between the orientation factor $\alpha$ and the disparity parameters of fibers $\left(\sigma_{A z}, \sigma_{E l}\right)$ are shown in Table 2. The first column stands for the perfect uniform orientation of fibers in $3 \mathrm{D}$, the second column reproduces the case of uniform planar orientation of fiber and the last column is the case when fibers are considered perfectly aligned in direction $z$.

Table 2: Correspondence between parameters $\left(\sigma_{E l}, \sigma_{A z}\right)$ and orientation factor $\alpha_{z}\left(\right.$ for $\left.\mu_{A z}=\mu_{E l}=0\right)$

\begin{tabular}{lccc}
\hline uniform orient. in & $3 \mathrm{D}$ & $2 \mathrm{D}$ & $1 \mathrm{D}$ \\
$\alpha_{z}$ & 0.500 & 0.637 & 0.999 \\
\hline$\sigma_{A z}$ & 15.81 & 100.00 & 0.03 \\
$\sigma_{E l}$ & 15.81 & 0.02 & 0.03 \\
\hline
\end{tabular}

\subsection{Hardening behavior}

The elastic and hardening behavior are described by the following equation:

$$
\boldsymbol{\sigma}=\mathbb{C}(\epsilon): \varepsilon,
$$

where $\mathbb{C}$ is the fourth order stiffness tensor, $\epsilon$ stands for the anisotropic damage parameter, $\sigma$ and $\varepsilon$ are respectively the stress and strain tensors. The stiffness tensor $(\mathbb{C})$ is derived considering the cracked material as a perfectly elastic cementitious matrix containing inclusions with no rigidity and playing the role of cracks. The matrix and the inclusions are homogenized by the means of the Mori-Tanaka homogenization scheme, which takes into account the interaction of inclusions within the matrix as follows:

$$
\mathbb{C}=\mathbb{C}_{0}:\left(\mathbb{I}+\frac{4}{3} \pi\left(\epsilon-\epsilon_{0}\right) \mathbb{T}(\mathbf{n})\right)^{-1}
$$

where constant $\epsilon_{0}$ represents the initial isotropic damage threshold, we note that $\epsilon \geq \epsilon_{0}$ is always true and $\mathbb{I}$ is the fourth order identity tensor. The inclusions are considered as a family of parallel penny-shaped cracks with orientation $\mathbf{n}[18]$ and are mathematically modeled by the fourth order tensor $\mathbb{T}(\mathbf{n})$. The crack orientation is always collinear with the principal maximum strain direction : only mode I fracture is modeled. The tensor $\mathbb{C}$ is therefore a secant transverse-isotropic stiffness matrix.

Within the energy-based framework of Linear Elastic Fracture Mechanics (LEFM), the model considers the external loads and the fiber bridging forces as external forces applied on an elastic medium. For this loading scheme, the total stress intensity factor is the difference (considering the convention of the load direction with respect to the work-conjugate displacement) of the stress intensity factor of each load. In using Irwin relationship $\left(G=K^{2} / E\right)$, the energy release rate can be formulated as [19, Sec. 3.5.2]:

$$
f=\mathcal{G}_{A}+\mathcal{G}_{B}-2 \sqrt{\mathcal{G}_{A} \mathcal{G}_{B}}-G_{C},
$$

where

$$
\mathcal{G}_{A}=-\frac{\partial \Psi}{\partial \epsilon}=-\frac{1}{2} \varepsilon: \frac{\partial \mathbb{C}}{\partial \epsilon}: \varepsilon
$$

is the energy release rate of external loads: it is defined as the derivative of the free energy $\Psi$, by damage parameter $\epsilon \equiv \mathcal{N}_{c} a^{3}$, defined by Budiansky, and assuming inclusions of pennycracked shape of radius $a$;

$$
\mathcal{G}_{B}=\frac{\mathrm{d} W(a)}{\mathrm{d} a}=\mathcal{N}_{c} \frac{\partial a}{\partial \epsilon} \frac{\partial W(\epsilon)}{\partial \epsilon}
$$

is the energy release rate which takes into account the contribution of fibers [9], where $W(a)$ is the amount of energy dissipated by fibers being extracted from the matrix by a penny cracked-shaped of radius $a$. Assuming the maximum opening of the crack $(w)$ is inferior to $\frac{L_{f}}{2}$, the amount of energy is :

$$
W(a)=2 \pi \int_{0}^{a} \int_{0}^{w} \sigma_{c}(w) r \mathrm{~d} w \mathrm{~d} r .
$$

And

$$
G_{c}=\frac{2 \pi}{3}\left(\frac{\mathcal{N}_{c}}{\epsilon}\right)^{\frac{1}{3}} G_{f}
$$

is the equivalent fracture energy of the representative element volume written as a function of the damage parameter $(\epsilon), G_{f}$ is the specific 
fracture energy of the matrix and $\mathcal{N}_{c}$ is the number of cracks per unit volume.

The evolution of damage is controlled by Eq. 10 thanks to the Kuhn-Tucker conditions :

$$
f(\epsilon) \leq 0 ; \quad f(\epsilon) \mathrm{d} \epsilon=0 ; \quad \mathrm{d} \epsilon \geq 0 .
$$

The resolution of this system, for a given strain history along with the consistency condition $\mathrm{d} f=0$, gives the stress-strain material law of the material beyond the elastic part.

\subsection{Softening rotating crack model}

Once the maximum cohesive stress $\left(\sigma_{c, \text { max }}\right)$, computed from Eq. 4, is reached, a softening behavior follows based on $F=F_{p}$. The cohesive stress $\left(\sigma_{c}\right)$ controls the softening part of the constitutive law expressed by:

$$
\boldsymbol{\sigma}=\mathbb{C}\left(\epsilon_{\max }\right):(\boldsymbol{\varepsilon}-\underbrace{\mathbf{p}_{1} \otimes \mathbf{p}_{1} \varepsilon_{n n}^{c}}_{\varepsilon_{c}}),
$$

where $\mathbb{C}\left(\epsilon_{\max }\right)$ is the stiffness tensor at peak, $\varepsilon$ the total strain tensor, $\mathbf{p}_{1}$ is the direction of maximum principal tensile stress, $\mathbf{n}$ is the crack normal, and $\varepsilon_{n n}^{c}$ the normal crack strain [20]. The model is implemented according to a classical rotating crack model, i.e., the crack orientation is allowed to evolve during loading. Unit vector $\mathbf{n}$ is constrained to be collinear with $\mathbf{p}_{1}$ so that no shear can be developed on the crack plane.

To avoid well-known issues of mesh size dependency, the stress-strain law is made dependent on the mesh size to guarantee that the density energy dissipation is constant [19]. Hence, the cracking strain $\left(\varepsilon_{c}\right)$ is obtained thanks to the inverse of the cracking law:

$$
\varepsilon_{n n}^{c}=\frac{w}{h_{c}} \frac{h_{r e f}}{L_{0}}=\frac{\sigma_{c}\left(s_{n n}\right)^{-1}}{h_{c}} \frac{h_{r e f}}{L_{0}}
$$

where $s_{n n}$ is the maximum principal stress, $L_{0}$ is a characteristic length, $h_{c}=\sqrt[3]{V}$ is the regularization parameter with $V$ being the element volume and $h_{\text {ref }}$ a constant equal to $1 \mathrm{~mm}$. To ensure an efficient regularization with this definition, the mesh should be composed of cubes as far as possible. The model is implemented in the open source Code_Aster finite element code [21]. Futher details are given in [24].

\section{Model Validation}

The model was validated using uniaxial tensile tests on UHPFRC [7], where fibers are oriented along a preferential direction by constraining fibers with steel molds. The specimens were unnotched with a dimension of $600 \times 50 \times 20 \mathrm{~mm}$ [7].

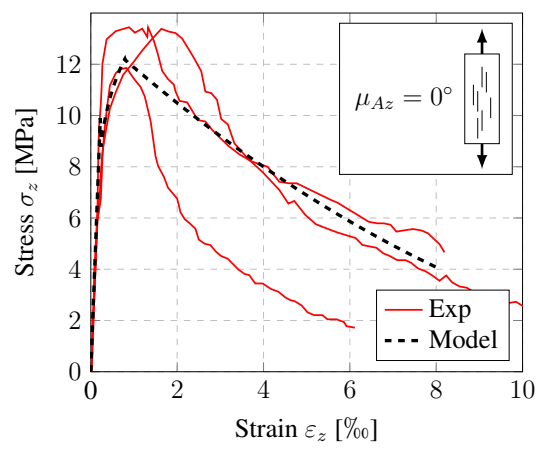

Figure 3: Results of calibration, after [7]

In a first step, model parameters have been calibrated in using results from the case where fibers are aligned to the loading direction (see Fig. 3). The elastic matrix modulus $\left(E_{m}\right)$ is used to capture the elastic phase, the bond strength of fiber-matrix interface $\left(\tau_{0}\right)$ to calibrate the maximal tensile stress, the initial crack damage parameter $\left(\epsilon_{0}\right)$ to set the strain corresponding to maximal stress, the specific fracture energy of the matrix $\left(G_{f}\right)$ to set the elastic limit, the characteristic length $\left(L_{0}\right)$ to capture the softening curve and finally the disparity of fiber orientation $\left(\sigma_{A z}=\sigma_{E l}\right)$ is chosen considering the crack interface profile. Some other parameters are given: volume of fibers $\left(v_{f}\right)$, fiber length $\left(L_{f}\right)$, fiber elastic modulus $\left(E_{f}\right)$ and fiber diameter $\left(\phi_{f}\right)$. Table 3 reports all the parameters values.

Table 3: Fibers and matrix model parameters

\begin{tabular}{rlrl}
\hline$v_{f}$ & $=2.7 \%$ & $E_{f}$ & $=210 \mathrm{GPa}$ \\
$L_{f}$ & $=13.0 \mathrm{~mm}$ & $\phi_{f}$ & $=0.20 \mathrm{~mm}$ \\
$\nu$ & $=0.18$ & $a_{0}$ & $=1 \mathrm{~mm}$ \\
\hline$E_{m}$ & $=45 \mathrm{GPa}$ & $f_{t}$ & $=8.0 \mathrm{MPa}$ \\
$\sigma_{E l, A z}$ & $=10.0$ & $\mu_{E l}$ & $=0^{\circ}$ \\
$L_{0}$ & $=350 \mathrm{~mm}$ & $\tau_{0}$ & $=8.0 \mathrm{MPa}$ \\
$\mathcal{N}_{c}$ & $=0.0003 \mathrm{cracks} / \mathrm{mm}^{3}$
\end{tabular}


In a second step, we compared the simulated and experimental stress-strain curves for other experimental cases where the preferential fiber inclination with respect to load directions was varied [7]. In the model, we only changed the angle of the fiber orientation $\left(\mu_{A z}\right)$. Figure 4 shows that the model globally captures the experimental results and therefore the effect of fiber orientations.
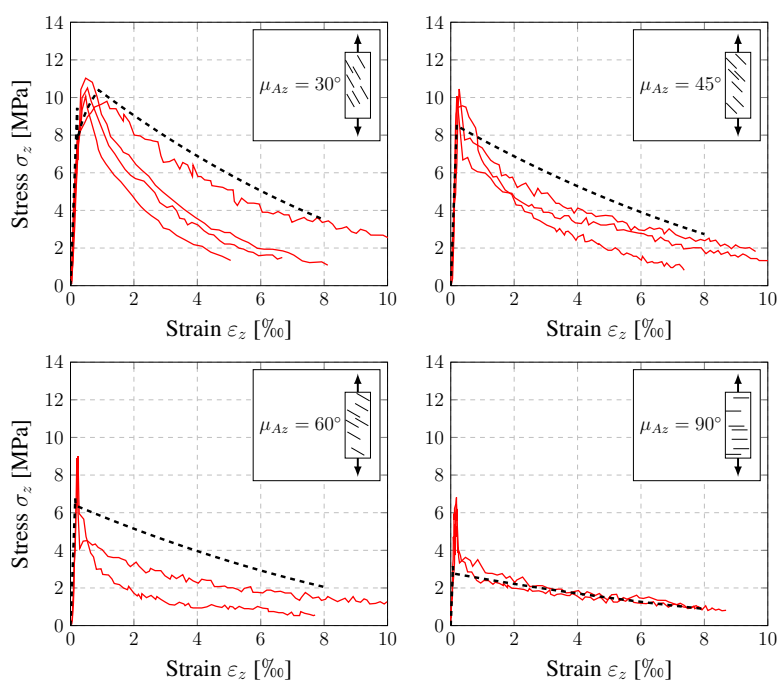

Figure 4: Validation for different orientation of fibers, after [7]

Fracture energy and maximal stress are well estimated whereas elastic limit stress is slightly overestimated. This means that the fracture energy of the matrix, in terms of damage, see Eq. 14, can be improved using different crack shapes or other definitions of damage parameter.

\section{FEM ANALYSIS OF BENDING TESTS}

To verify the ability of the model to accurately reproduce the behavior of UHPFRC reinforced structures, we first carried out four point bending tests on $40 \times 100 \times 1400 \mathrm{~mm}$ beams made of UHPFRC with $1 \%$ and $2 \%$ volumetric fiber content [22]. Half of them were reinforced with an $8 \mathrm{~mm}$ diameter steel bar (Fig. 5). The loading control of the test was accomplished by completing the test in a servohydraulic load frame. The control signal for all tests was the stroke with the imposed rate equal to $0.3 \mathrm{~mm}$ per minute before crack localization and, then, increased to $0.6 \mathrm{~mm} / \mathrm{min}$ after crack localization. The mid-span deflection was measured on one beam side by a LVDT with a range of about $50 \mathrm{~mm}$, supported by an aluminum yoke hanged on the beam supports so that support settlement was eliminated. The other side of beams was used for digital image correlation.

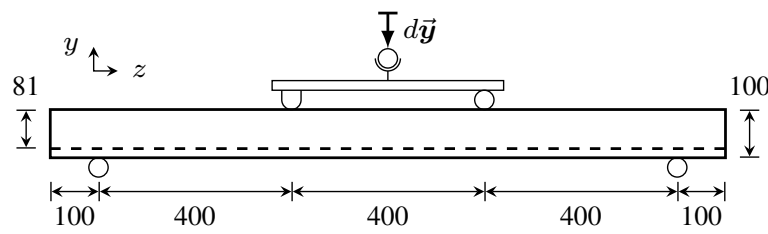

Figure 5: Experimental setup [22]

Additional specimens were cast to saw prisms from them and to measure the real fiber orientation at the corresponding position of the macroscopic localized crack by means of $\mathrm{X}$ rays tomography [23]. The analysis was performed over four heights levels for either $1 \%$ and $2 \%$ samples without rebars; as an example, Fig. 6 shows the first top level with $1 \%$ fiber ratio (all other cases are presented in [24]).

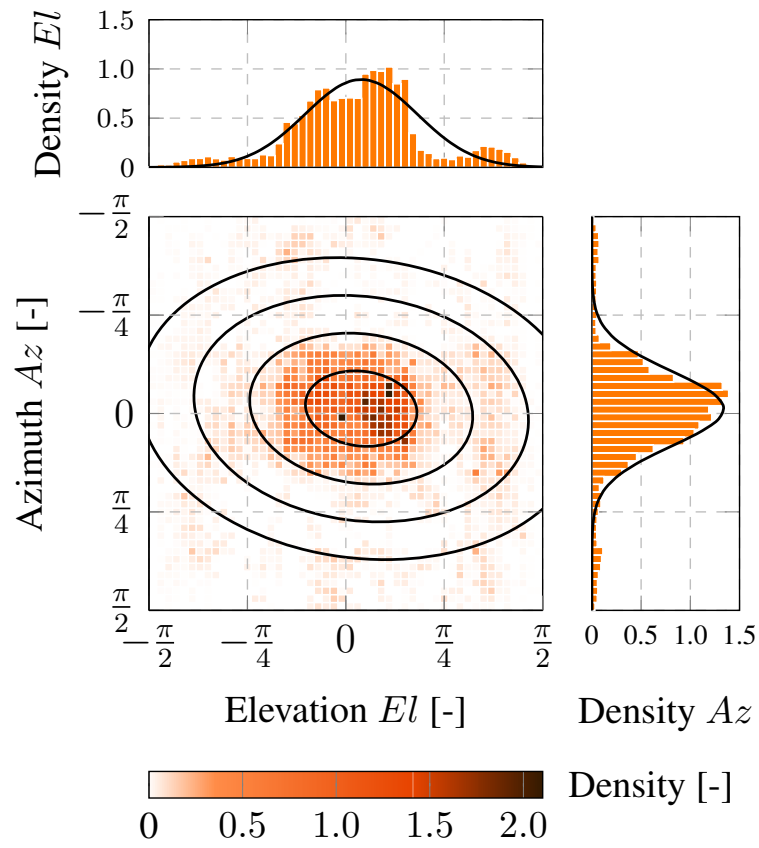

Figure 6: Fiber orientation on top of the beam resulting from tomography analysis 
The 1D and 2D histograms show the dispersion of fiber orientation with respect to the azimuth and the elevation. From the tomography analysis, we were able to obtain the fiber orientation coefficient from top to bottom of the beam: $0.88,0.89,0.93$, and 0.95 . These values show that fibers are more aligned in the bottom of the mold rather than on top, which is consistent with wall effects. Moreover, the tomography analysis revealed a segregation of fibers varying from top to bottom : $v_{f}=0.7 \%, 0.9 \%$, $1.1 \%$, and $1.3 \%$. This information was used as input data for the model (similar dispersions were obtained for $2 \%$ of fibers [24]).

For the simulation of the four point bending tests, we used a mesh of 3520 linear hexaedrons elements. We reproduced the difference in displacement of East and West cylinders thanks to data from digital image correlations. In order to initiate the localized crack at the correct position, we specified to the closest element a fiber volume of 3\% fewer fibers than neighboring elements (see Fig. 7).

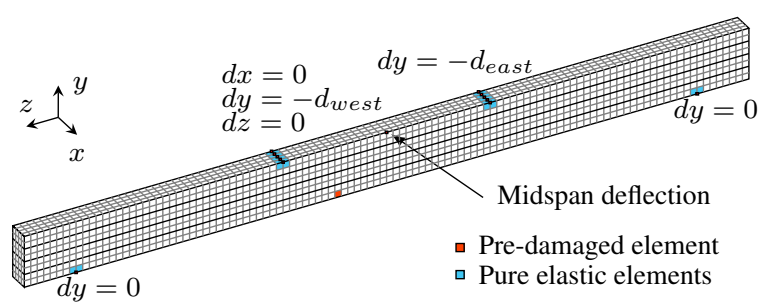

Figure 7: Mesh and group of mesh used to model the four points bending test

The bond strength of fiber-matrix interface $\left(\tau_{0}\right)$ was calibrated for both $1 \%$ and $2 \%$ volumetric fiber content ; the mean values (on three and four beams) are respectively: $12.56 \mathrm{MPa}$ and $8.82 \mathrm{MPa}$. The values for $2 \%$ of fibers are lower because it takes into account the group effect of fibers; those results are very close to literature $[25,26]$. Concerning the characteristic length $\left(L_{0}\right)$, one beam was simulated considering the real displacement of East and West cylinders for each of the $1 \%$ and $2 \%$ fiber volumetric cases: we respectively obtained $1.0 \mathrm{~mm}$ and $1.3 \mathrm{~mm}$.
The material parameters were calibrated on experimental data as shown in Fig. 8. The approximate discrete position of the simulated crack with the corresponding simulated cylinders displacement produced some time steps that did not converge even though fifteen Newton iterations were enabled; these outlier results correspond to dots out of the trend of the global beam behavior. For those time steps, the global residual did not diverge but the convergence was very slow or oscillating. This was certainly due to multiple integration points changing state from opening to decreasing crack width. Nevertheless, the model makes it possible to capture experimental data when adjusting material parameters $\left(\mathcal{N}_{c}\right.$ for the hardening length, $\tau_{0}$ for the maximum resistance and $L_{0}$ for the softening curve).

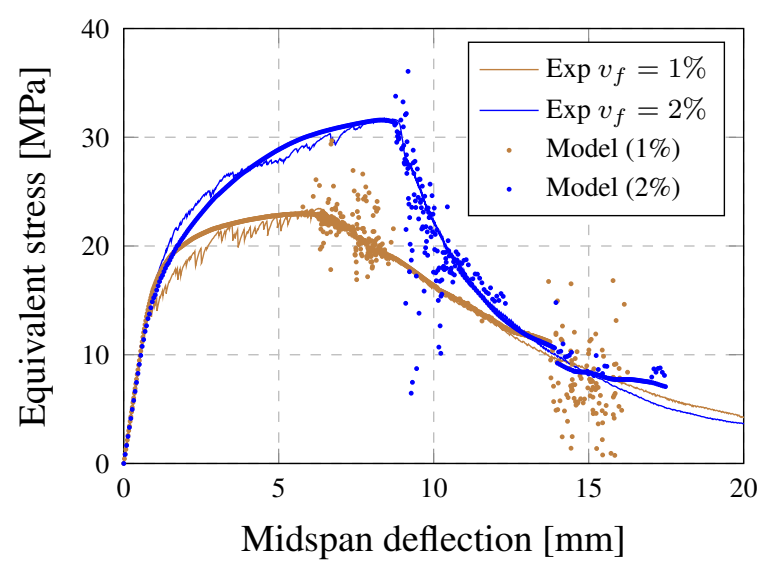

Figure 8: Calibration on experimental results

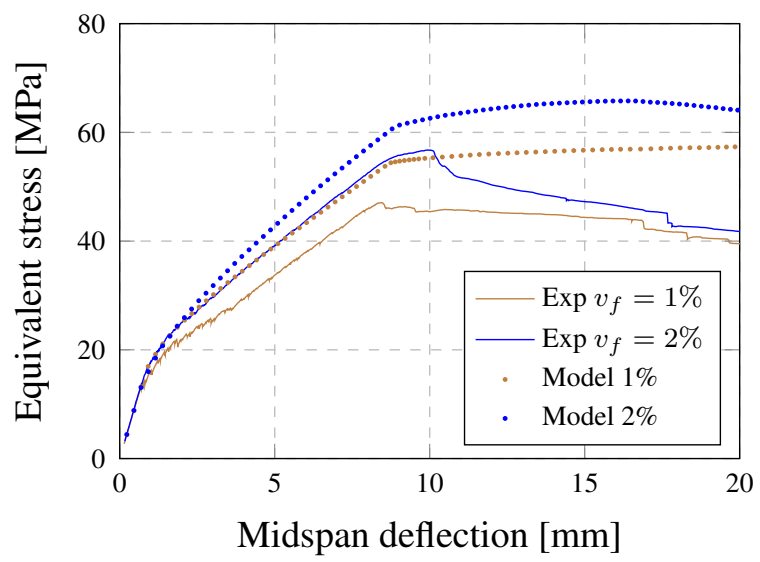

Figure 9: Modeling the reinforced beam 
Considering the obtained material parameters, we also simulated the beams containing the reinforced bars. The same mesh was used with the addition of 1D linear isotropic strain hardening bar elements with von Mises yield criterion to model the reinforcement. Figure 9 shows that the simulation overestimates the beam resistance.

This can be attributed to the modeling of the interface between UHPFRC and the rebar which was considered as perfectly anchored. The difference of strength between simulation and the experimental results is smaller for UHPFRC with $v_{f}=2 \%$ because the assumption of perfect bonding between UHPFRC and rebar is less irrelevant. Moreover, in the simulation, the fiber orientation and distribution have been considered similar to beams with and without rebars. Nevertheless, the fiber orientation is disturbed by the reinforcement.

\section{CONCLUSIONS}

A model has been developed combining fracture-micromechanics and smeared rotating cracks to reproduce the behavior of UHPFRC under tension. The constitutive law is softening and eventually hardening depending on material parameters. The model takes into account fiber orientation and distribution, fiber volume and geometry, matrix fracture energy, and fiber-matrix interface characteristics. The orientation of fibers is modeled by a bivariate $\pi$ periodic normal-like probability density distribution which makes it possible to simulate various casting process. This parameter can easily be linked to the traditional orientation factor.

The resulting model is able to reproduce four point bending tests of rectangular cross-section UHPFRC beams, with different fiber volumetric contents. The preliminary FEM analysis is encouraging as a refined tool for predicting the maximum load considering fibers effect on the global behavior, and needs to be extended to grasp more accurately the bar reinforcement effect.

\section{ACKNOWLEDGEMENT}

Partial financial support from Lafarge and CRSNG are gratefully acknowledged. The authors particularly thank D. Corvez and ${ }^{\dagger} \mathrm{G}$. Chanvillard from Lafarge for fruitful discussion.

\section{REFERENCES}

[1] NF P18-470. 2016. Bétons fibrés à ultra-hautes performances. Spécification, performance, production et conformité. Afnor, $79 \mathrm{pp}$.

[2] NF P18-710. 2016. Calcul des structure en béton : règles spécifiques pour les bétons fibrés à ultra-hautes performances, éléments complémentaires. Afnor, 137 pp.

[3] fib MC 2010. The fib Model Code for Concrete Structures. Ernst \& Sohn.

[4] Simon, A., Corvez, D., and Marchand, P. 2013. Feedback of a ten years assessment of fibre distribution using factor concept. In Toutlemonde, F. and Resplendino, J. (eds), UHPFRC 2013, pp. 669-678, Marseille, France. RILEM Pro087.

[5] Laranjeira, F., Aguado, A., Molins, C., Grünewald, S., Walraven, J., and Cavalaro, S. 2012. Framework to predict the orientation of fibers in FRC: A novel philosophy. Cement and Concrete Research, 42(6):752 - 768.

[6] Martinie, L. and Roussel, N. 2011. Simple tools for fiber orientation prediction in industrial practice. Cement and Concrete Research, 41(10):993 - 1000.

[7] Bayard, O. 2003. Approche multi-échelles du comportement mécanique des bétons à ultra hautes performances renforcés par des fibres courtes. PhD thesis, École Normale Supérieure de Cachan, France.

[8] Bernier, G. and Behloul, M. 1996. Effet de l'orientation des fibres sur le comportement des BPR. In $2 e$ coll. inter. francophone sur les bétons renforçés de fibres métalliques, pp. 233-240. Revue française de génie civil.

[9] Lin, Z. and Li, V. C. 1997. Crack bridging in fiber reinforced cementitious com- 
posites with slip-hardening interfaces. $J$. Mech. Phys. Solids (UK), 45(5):763 - 87.

[10] Bao, G. and Song, Y. 1993. Crack bridging models for fiber composites with slipdependent interfaces. J. Mech. Phys. Solids, 41(9):1425 - 1444.

[11] Wuest, J. 2007. Comportement structural des bétons de fibres ultra performants en traction dans des éléments composés. $\mathrm{PhD}$ thesis, EPFL.

[12] Naaman, A. E. and Shah, S. P. 1976. Pullout mechanism in steel fiber-reinforced concrete. Journal of the Structural Division, 102(8):1537 - 1548.

[13] Maage, M. 1977. Interaction between steel fibers and cement based matrixes. Matériaux et Constructions, 10(59):297 301.

[14] Sorelli, L., Ulm, F.-J., and Toutlemonde, F. 2007. Fracture stability and micromechanics of strain hardening cementitious composites. FraMCoS-6, 3, pp.14031411, Catania, Italy.

[15] Guénet, T., Sorelli, L., Corvez, D., Bastien, J., Toutlemonde, F., Ferrier, E., and Michel, L. 2013. Analysis of a UHPFRC footbridge with a deck slab under bending by a novel fracturemicromechanics fem model. In Toutlemonde, F. and Resplendino, J. (eds), UHPFRC 2013, pp. 519-528, Marseille, France. RILEM Pro087.

[16] Delsol, S. and Charron, J.-P. 2013. Numerical modeling of UHPFRC mechanical behavior based on fibre orientation. In Toutlemonde, F. and Resplendino, J. (eds), UHPFRC 2013, pp. 679-688, Marseille, France. RILEM Pro087.
[17] Kooiman, A. G. 2000. Modelling Steel Fibre Reinforced Concrete for Structural Design. PhD thesis, Delft University.

[18] Dormieux, L., Kondo, D., and Ulm, F.-J. 2006. Microporomechanics. Wiley.

[19] Bažant, Z. and Planas, J. 1998. Fracture and size effect in concrete and other quasibrittle materials. New directions in civil engineering. CRC Press.

[20] Jirásek, M. 2012. Modeling of Localized Inelastic Deformation - Lecture notes. Czech Technical University in Prague.

[21] Abbas, M. 2013. Introduction to Code_Aster. EDF R\&D.

[22] Duhamel-Labrecque, Y. 2016. Étude de la microfissuration, capacité rotationnelle et ductilité des poutres en bfup par analyse d'images. Master's thesis, Univ. Laval.

[23] Couprie, M., Meulenyzer, S., Salem, M., Talbot, H., and Pourcel, F. 2014. Fibre analysis in $3 \mathrm{~d}$ materials and process validation on artificial data. Journal of $\mathrm{Mi}$ croscopy, 255(2):78-88.

[24] Guénet, T. 2016. Modélisation du comportement des Bétons Fibrés à Ultra-hautes Performances par la micromécanique : effet de l'orientation des fibres à l'échelle de la structure. $\mathrm{PhD}$ thesis, Univ. Laval and Univ. Paris-Est.

[25] Máca, P., Sovják, R., and Konvalinka, P. 2014. Mix design of UHPFRC and its response to projectile impact. International Journal of Impact Engineering, 63:158 163.

[26] Yoo, D.-Y., Shin, H.-O., Yang, J.-M., and Yoon, Y.-S. 2014. Material and bond properties of Ultra High Performance Fiber Reinforced Concrete with micro steel fibers. Composites Part B: Engineering, 58:122 - 133 . 